\title{
Proton therapy for early stage prostate cancer: is there a case?
}

This article was published in the following Dove Press journal:

OncoTargets and Therapy

9 September 2016

Number of times this article has been viewed

\section{Tabitha Y Chan \\ Poh Wee Tan \\ Johann I Tang}

Department of Radiation Oncology, National University Cancer Institute, Singapore
Correspondence: Tabitha $Y$ Chan Department of Radiation Oncology, National University Cancer Institute, 5 Lower Kent Ridge Road, Singapore 119074

$\mathrm{Tel}+656772838$ ।

Fax +65 67342986

Email tabitha_chan@nuhs.edu.sg
Abstract: Proton-beam therapy (PBT) for prostate cancer has been in used for several decades, with its technique evolving significantly over this period. A growing number of centers now routinely utilize pencil-beam scanning as an advanced technique of PBT. Interest and controversy concerning its use have recently come under scrutiny. While the past decade has produced an assemblage of evidence suggesting that PBT is safe and effective for early stage prostate cancer, it is still unknown whether the theoretical dosimetric advantages of PBT translate into meaningful clinical improvements over routine intensity-modulated radiation therapy, which is commonly used for these patients. Outcomes from early trials using whole courses of PBT have shown mixed results when compared with routine intensity-modulated radiation therapy. Therefore, randomized trials comparing these two techniques should be undertaken, as this would help in defining the role of PBT for this patient group. This article aims to describe the basics of PBT, review the reasons for the growing interest in PBT, review the evidence for PBT, review the controversy surrounding PBT, and inquire about PBT's future in the treatment of prostate cancer, with attention to its physical properties, comparative clinical and cost-effectiveness, and advances in its delivery.

Keywords: proton beam, radiation, prostate cancer, clinical outcomes, controversies, future direction

\section{Introduction}

External-beam radiation therapy (EBRT) remains one of the primary treatment modalities for early stage prostate cancer. ${ }^{1}$ With advancement in technology, EBRT has evolved from a conventional technique typically delivered by means of a four-field technique to intensity-modulated RT (IMRT), ${ }^{1}$ which is used routinely worldwide. ${ }^{2,3}$ IMRT is a highly effective treatment option with a well-defined toxicity profile. ${ }^{3}$ Continuous improvements in radiation planning and delivery have allowed dose escalation to the prostate while minimizing toxicity to adjacent normal structures, termed as organs at risks (OARs).

Meanwhile, the technology of proton-beam therapy (PBT) has similarly improved. ${ }^{4}$ Its unique physical dose-deposition properties to avoid normal tissue give an advantageous dose distribution, and growing experience with PBT makes it an attractive prostate cancer treatment.

However, mixed opinions derived from results reported by nonrandomized studies, ${ }^{5-7}$ the lack of randomized data comparing PBT to modern photon-based therapy in the treatment of early stage prostate cancer, and the increased cost compared to other forms of treatment have resulted in PBT becoming a source of controversy. ${ }^{8-10}$

Therefore, this article aims to compare the basics of PBT with EBRT, review the evidence for and against PBT, and inquire about PBT's future in the treatment 
of prostate cancer, with attention to its physical properties, comparative clinical and cost-effectiveness, and advances in delivery.

\section{Comparing the basics of PBT with EBRT}

EBRT is the external delivery of radiation. It often uses photons and is the most widely used type of RT. ${ }^{11}$ Photons are massless and uncharged, ${ }^{12}$ while protons are massive, positively charged subatomic particles. ${ }^{13}$ The relative biological effectiveness (RBE), a measure employed in the field of radiation oncology to compare the effects of different forms of ionizing radiation on living tissue, of photons and protons are similar. ${ }^{14}$ Therefore, it is not the biologic properties but the physical properties of protons that confer a dosimetric advantage and make using PBT as a source of therapeutic radiation attractive. This physical property results in the majority of energy deposited at the end of their range at a sharp maximum known as the Bragg peak. Radiation dose then falls off rapidly beyond the Bragg peak. This essentially allows no exit dose and a significantly reduced dose beyond the target volume. The beam energy determines the position of the Bragg peak. An individual Bragg peak covers only a few millimeters and is too narrow to cover any tumor of realistic dimensions. Therefore, multiple beams of differing energies are combined and modulated to create staggered depths of penetration known as the "spread-out Bragg peak" (SOBP) to encompass uniformly the entire therapeutic target. ${ }^{15}$

Comparatively, photons deposit their biologically effective dose in a continuous linear fashion. This results in some dose received in the beam's path beyond the target. ${ }^{16}$ Furthermore, a megavoltage photon beam typically deposits its maximum energy within just a few centimeters of its entrance into the body. ${ }^{16}$ Therefore, deep-seated tumors, such as in the prostate, often require multiple beams to maximize conformity and avoid overdosing superficial structures and surrounding normal tissues. As such, the unique properties of protons confer a theoretical advantage in delivering higher doses of radiation to a given target while simultaneously limiting collateral damage to normal tissues that would otherwise be caught within the entrance and exit paths of a photon beam. This is particularly relevant to the prostate, as the gland itself resides deep within the pelvis and is in intimate contact with several dose-limiting proximal OARs, such as the bladder and rectum. ${ }^{17}$ Furthermore, variations in rectal and bladder filling affect the position of the prostate. These have important implications for treatment planning.

\section{Dosimetric considerations}

Due to technological advances in the past few decades, it is important to frame any comparison between PBT and photon therapy in the context of contemporary techniques. Currently, PBT can be delivered by two methods. The older method uses a three-dimensional (3-D) conformal technique where passively scattered proton beams of differing energies are combined to form an SOBP that fully encompasses the target. The most common beam arrangement uses two opposed lateral beams, and customized apertures and compensators are fabricated to shape the field and alter the dose-depth profile to conform the SOBP to the actual tumor better.

The second newer method utilizes proton pencil-beam scanning (PBS), which allows more conformal and complex "IMRT-like" distribution. This is known as intensity-modulated proton therapy (IMPT) with a greater ability to conform the dose to irregularly shaped target. ${ }^{18}$ Since IMRT is now the most common photon-based EBRT technique used for the treatment of prostate cancer, it serves as the reference in modern comparative studies..$^{19,20}$

Dosimetric studies evaluating the relative advantages of PBT and IMRT have been conducted at several centers. These studies compared passively scattered PBT and IMRT in their ability to spare proximal OARs. ${ }^{5,6}$ Trofimov et al compared PBT plans utilizing two opposed lateral beams to IMRT plans utilizing seven coplanar beams in ten patients with early stage prostate cancer at Massachusetts General Hospital. ${ }^{5}$ The planning target volume (PTV) was expanded by $10 \mathrm{~mm}$ from the clinical TV to account for intrafractional motion and setup uncertainties. After adjustment for RBE, where a dose of 79.2 Gy was prescribed to the prostate, PBT significantly reduced the volume of bladder and rectum irradiated, especially in areas receiving low-to-moderate dose exposure (ie, normal tissue exposure in the $<30$ Gy RBE range). However, IMRT achieved greater reduction for the bladder in the high-dose region (ie, normal tissue exposure in the 50-75 Gy RBE range), while no difference in the rectal volume was noted at these doses. ${ }^{5}$ Vargas et al conducted a similar dosimetric study in Florida. ${ }^{6}$ However, unlike Trofimov et al, who gave a uniform expansion of $10 \mathrm{~mm}$ for the PTV, ${ }^{5}$ Vargas et al expanded the clinical TV by $5 \mathrm{~mm}$ and $8 \mathrm{~mm}$ in the axial and craniocaudal dimensions, respectively, to create the PTV. ${ }^{6}$ Furthermore, instead of two opposed lateral beams for PBT, PBT gantry angles were optimized to utilize lateral-oblique beams with small posterior angles. This improved PTV dose distribution minimized rectal and bladder doses. The added merit of the Vargas et al study was that both PBT and IMRT plans were developed using 
the same computed tomography data set of each patient. ${ }^{6}$ Similarly to Trofimov et al, PBT achieved significant bladder and rectum dose reduction in the low-dose region (as denoted by $<35$ Gy RBE range). ${ }^{5,6}$ Unlike Trofimov et al, where there was no difference in the rectal volume noted at high doses, rectal volumes in the medium-high dose range of 35-80 Gy (RBE) were significantly lower in Vargas et al. ${ }^{5,6}$ However, the University of Texas ${ }^{7}$ conducted a separate analysis with similar 5-8 mm anisotropic margins for ten patients treated with opposed lateral beams, and found no significant difference in the rectal and bladder volumes irradiated to $40 \mathrm{~Gy}$ (RBE) or higher for IMRT or PBT.

These studies demonstrate a reduction in low-dose exposure with PBT with similar or even higher volumes of OARs exposed to high doses. This has resulted in mixed opinions about the dosimetric advantage of PBT.

The merits of PBT are the potential reduction in radiationinduced morbidities of proximal OARs abutting the prostate due to dose reduction and significant reduction in whole-body radiation exposure. This reduction in whole-body radiation exposure has significant potential implications. Symptoms of hypogonadism and reduced serum testosterone, due to scattered dose to the testicles, are possible side effects of IMRT. ${ }^{21-23}$ With significant reduction in whole-body exposure associated with PBT, these effects may be lessened. ${ }^{24,25}$ Although rare, a long-term side effect associated with normal tissue exposure is the increased risk of secondary malignancies. Given this long latent period, the absolute risk of men above 70 years old is very small. However, this could be of greater importance to younger men. The literature has also demonstrated as much as a $40 \%$ reduction in risks of secondary malignancies related to radiation exposure when PBT is used. ${ }^{26-28}$ Although these studies demonstrate a reduction in low-dose exposure with PBT, the higher volumes of OARs exposed to high doses should be duly considered. To date, the relative contribution of high- versus moderate-dose regions to acute and late toxicity remains a topic of contention. ${ }^{29-35}$

\section{Theoretical causes of concern}

While these studies affirm the dosimetric advantage conferred by the unique properties of PBT, uncertainties pertinent to the treatment of deep-seated targets, such as the prostate, do exist. ${ }^{36}$

The sharpness of the Bragg peak is consistent within the first $10 \mathrm{~cm}$ of tissue. However, "range uncertainty" at depths greater than $10 \mathrm{~cm}$ and the development of a lateral penumbra at the distal end of the beam results in dose blurring.
Therefore, higher-than-anticipated doses are potentially delivered to adjacent normal tissues. ${ }^{37}$ This is particularly relevant to prostate cancers, where the typical depth of the prostate within the body is approximately $20 \mathrm{~cm}$. Although there is no widely used method to confirm the proton range or that the SOBP encompasses the prostate in vivo, the algorithms used to calculate the doses appear accurate enough to raise any significant issues should sufficient compensation be used. To manage this range uncertainty for 3-D conformal PBT plans, diode radiation detectors embedded on the anterior surface of a rectal balloon could be used to record the radiation-dose rate at the distal penumbra of an anteriorly oriented proton field with millisecond resolution. This provides in vivo range verification and allows dosimetrists to minimize the required distal range-uncertainty margin. ${ }^{38}$

The dose distribution of the proton beam is sensitive to heterogeneous changes in tissues. This has significant impact on practical issues, such as uncertainty in patient setup and daily anatomic variation. Regarding patient setup, where laterally oriented beams are utilized, minute difference in femoral head rotations could significantly distort proton-dose distribution when the proton beam passes through the hips. Yoon et al studied the impact of inter- and intrafractional movement on target doses for patients treated with opposed lateral proton fields by calculating the dosimetric consequences of movement in each direction during and between fractions. ${ }^{39,40}$ They demonstrated that increasing movements were proportional to a decrease in target coverage. ${ }^{40}$ Differential effects of motion on IMRT versus PBT plans were more pronounced in the lateral direction, where a shift of $6 \mathrm{~mm}$ for a PBT plan was associated with a 9\% decrease in coverage compared to only $1 \%$ for the IMRT plan. ${ }^{40}$ This target-movement effect can be compensated by increasing the PTV margin and utilizing image-guidance verification. Even with image-guidance verification utilized to minimize errors in setup uncertainty, daily variations of in vivo parameters, such as tissue thickness, minute femur rotations, and uncertainty in estimating the proton-stopping power of different tissues, warrants the need for distal and proximal rangemargin and range-compensation expansions. ${ }^{41}$ When these margins were expanded, the volume of prostate not covered by the fractional prescription dose (as determined by a planning computed tomography data set) averaged less than 3\% daily, while more than $98 \%$ of the prostate received the total prescription dose over the treatment course without exceeding bladder and rectal constraints. ${ }^{42}$ However, this inevitably increases normal tissue exposure too. Therefore, this warrants strict adherence to reproducible immobilization during 
treatment, perhaps even more so than photon EBRT. ${ }^{40,41}$ Further uncertainty is also introduced by intrafraction organ motion, ${ }^{42}$ which is detrimental due to the deep dose depletion beyond the SOBP. ${ }^{40}$ The prostate position is dependent on the fullness of the bladder and rectum. To overcome this issue, fiducial markers with pretreatment localization and placement of rectal balloons to fix the prostate location have been successfully utilized. ${ }^{43}$

High-energy neutrons may be produced during PBT. This may negate some of the reduction in overall radiation exposure discussed earlier, as these neutrons have a particularly high $\mathrm{RBE} .{ }^{44}$ Other biological concerns include the concept that the RBE of protons may in fact increase beyond the Bragg peak. ${ }^{45}$ Therefore, in view of the theoretical nature of these physical and biological uncertainties, it is still debatable whether or not they translate into measurable clinical uncertainties that could impact toxicity.

\section{Reviewing the literature: efficacy, safety, and toxicity}

In all modalities of prostate radiation, toxicity primarily arises from exposure of normal tissues that lie in close proximity to the prostate. Rectal/bowel, bladder, and sexual dysfunction are the most common prostate radiationinduced toxicities. Budäus et al compiled a comprehensive review describing theses toxicities. ${ }^{46}$ Although the theoretical advantages of $\mathrm{PBT}$ are compelling, these are yet to be confirmed by randomized trials directly comparing photon IMRT with PBT. However, single-arm trials are available.

Loma Linda University Medical Center reported the first large single arm experience. ${ }^{47}$ Of the 1,255 patients studied, 731 received a combination of photon therapy and PBT to the prostate and pelvic lymph nodes and 524 received PBT alone to the prostate. Patients across all risk groups were included. The American Society for Therapeutic Radiology Oncology (ASTRO) consensus criteria were used. With a median follow-up of 62 months, the overall 5-year and 8 -year actuarial biochemical disease-free survival rates were $75 \%$ and $73 \%$, respectively, ${ }^{47}$ and are comparable to similar studies over the same time period, although the follow-up for these studies was short. ${ }^{48}$ Of greater importance in the Loma Linda trial is the significant improvement in treatment-associated toxicities. There were very low rates of Radiation Therapy Oncology Group grade 3 or higher morbidity: $1 \%$ genitourinary (GU) and $0.2 \%$ gastrointestinal (GI). ${ }^{47}$ To put these numbers in perspective, Vora et al found $6 \%$ GU and $1 \%$ GI grade 3 or higher morbidity when using
IMRT. ${ }^{49}$ This supports the theory that PBT is associated with reduced toxicities.

Massachusetts General Hospital and Loma Linda (Proton Radiation Oncology Group/American College of Radiology 95-09) conducted a multi-institutional randomized Phase III dose-escalation trial to evaluate the utility of PBT for prostate cancer, ${ }^{50}$ with 393 patients enrolled. A mixed photon/proton approach, using PBT for the boost (only the total dose of the boost differed by arm), demonstrated that a dose escalation to $79.2 \mathrm{~Gy}$ (RBE) instead of $70.2 \mathrm{~Gy}$ (RBE) had superior biochemical failure-free survival. Furthermore, patients receiving escalated doses experienced a low rate of acute and late grade 3 GI and GU toxicity (4\% and 3\%, respectively). Similar studies demonstrated similarly low rates of grade 3 toxicity. ${ }^{51,52}$ Therefore, this affirms that PBT allowed superior long-term cancer control without a significant increase in late morbidity. A quality of life (QoL) study was also conducted on these patients and found no significant differences in patient-perceived bowel, urinary, or sexual QoL between the arms. ${ }^{53}$

The PROG 95-09 trial aimed to determine if increasing radiation doses to men with early stage prostate cancer would improve clinical outcomes. ${ }^{50}$ Men with T1b-T2b prostate cancer and prostate-specific antigen $15 \mathrm{ng} / \mathrm{mL}$ were randomly assigned a total dose of either $70.2 \mathrm{GyE}$ (conventional) or 79.2 GyE (high). No patient received androgen-suppression therapy with radiation. All 393 men received 50.4 Gy delivered with photons in 1.8 Gy fractions to the prostate and seminal vesicles. Patients were randomized to receive a PBT boost of either 19.8 GyE or $28.8 \mathrm{GyE}$ in 1.8 Gy fractions. The median follow-up was 8.9 years. At 10 years, the ASTRO biochemical failure rates were $32.4 \%$ for conventional-dose and $16.7 \%$ for high-dose RT $(P<0.0001) ; 2 \%$ of patients in both arms experienced late grade $\geq 3$ GU toxicity, and $1 \%$ of patients in the high-dose arm experienced late grade $\geq 3$ GI toxicity. ${ }^{50}$ When compared to photon dose-escalation studies, this PBT dose escalation was achieved without significant increases in grade $\geq 3$ late GI or GU toxicities (Table 1)..$^{50,54-61}$

Post hoc analysis demonstrated no significant differences in patient-reported bowel, bladder, or sexual function between low- and high-dose arms at a median follow-up of 9 years. ${ }^{53}$ When compared to a similar cohort of patients treated with 3-D conformal radiation (although to a slightly lower dose), there was no significant difference in outcomes.

Similar analyses have been performed for patients receiving whole courses of PBT. In a multi-institutional prospective Phase II Japanese trial, ${ }^{62}$ patients received 74 Gy (RBE) for 


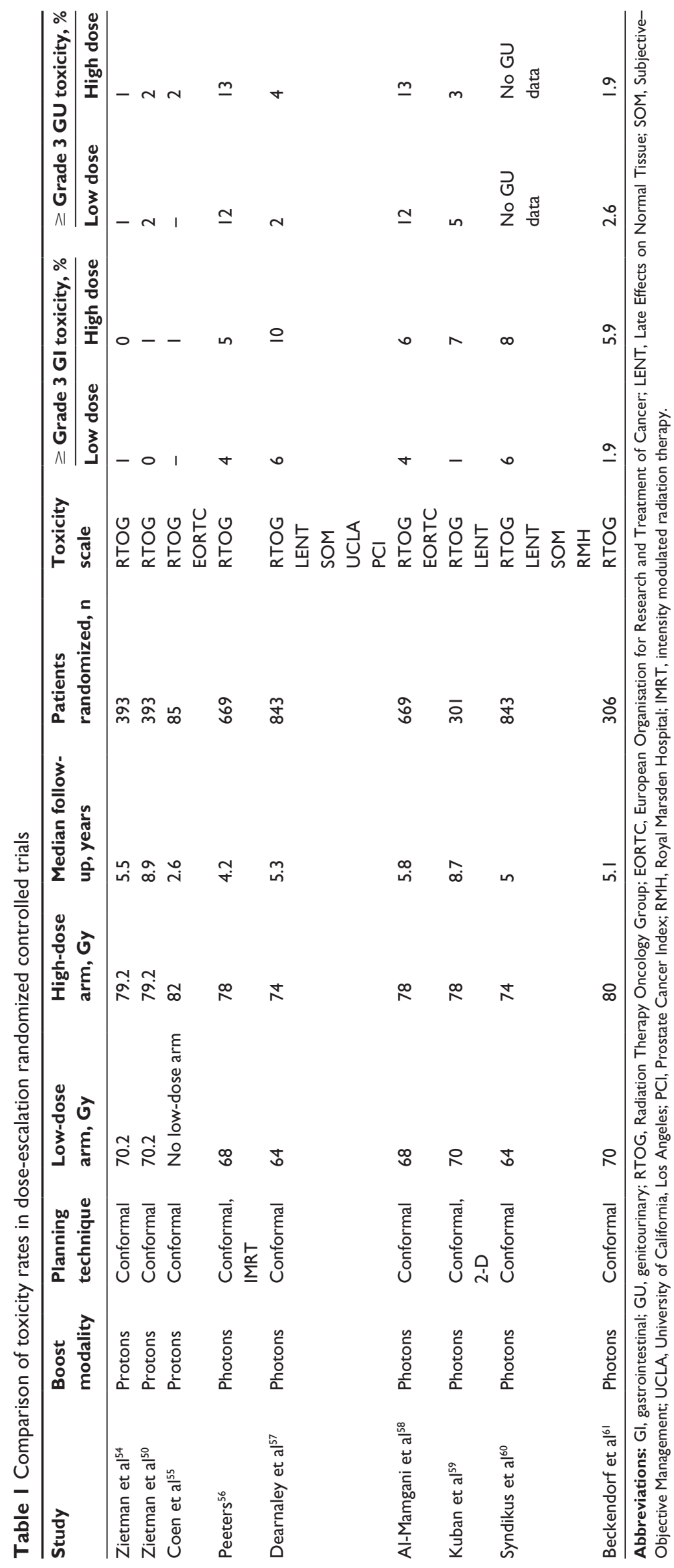


low- or intermediate-risk prostate cancer. Using the National Cancer Institute common toxicity criteria, acute grade 2 rectal and bladder toxicities occurred in $0.7 \%$ and $12 \%$ of patients, respectively. No patients experienced acute or late grade 3 rectal toxicity, and only $1 \%$ of patients had late grade 3 urinary toxicity. For patients with a follow-up of more than 2 years, the incidence of late grade 2 or greater rectal and bladder toxicity was $2 \%$ and $4 \%$, respectively. The most commonly reported rectal toxicity was bleeding, followed by pain and urgency. ${ }^{62}$

In 2012, Mendenhall et al had published the early outcomes of three prospective trials of PBT for low-, intermediate, and high-risk prostate cancer. ${ }^{63}$ Each risk group had a separate prescribed treatment plan. Only the high-risk group received androgen deprivation. The 2-year progression-free survival for the whole cohort was $99 \%$, whereas grade $3 \mathrm{GU}$ and GI toxicities were similar at 1.8\% (all were temporary) and $0.5 \%$, respectively. Although not directly comparable, these toxicity results favor PBT compared to 3-D conformal radiotherapy (3DCRT) or IMRT. ${ }^{63}$

Patient reported long-term QoL outcomes from a cohort of 95 men with localized prostate cancer treated with conformal protons and receiving a median dose of 78 Gy (RBE) were significant changes in incontinence, bowel, and sexual dysfunction, but not obstructive/irritative voiding dysfunction. ${ }^{64}$ When stratified by baseline functional category, only men with normal function prior to treatment had worsening score of incontinence and bowel dysfunction. For sexual dysfunction, there were significant changes in men with both normal and intermediate function, but not poor function. This study emphasized the importance of pretreatment functional status in estimating toxicity risk and using patient-reported outcomes to characterize the long-term sequelae of treatment.

Direct comparisons between photon therapy and PBT are difficult, due to differences in patient characteristics, dose, and treatment technique. However, toxicity and QoL outcomes are generally similar. Analyses comparing PBT toxicities to the low toxicity reported in modern IMRT techniques are most relevant. For example, Vora et al reported that in a cohort of 145 men receiving IMRT to a median dose of $75.6 \mathrm{~Gy}$, rates of grade $3 \mathrm{GI}$ and GU toxicity were $1 \%$ and $3 \%$, respectively. ${ }^{49}$ At a median of 5 years, $6 \%$ of patients had chronic grade 3 GI toxicity, while $1 \%$ had chronic grade 3 GU toxicity. ${ }^{49}$

In spite of the compelling theoretical advantage of PBT, recent results have questioned this benefit. ${ }^{2,29,65,66} \mathrm{~A}$ multiinstitutional study prospectively collected data on QoL to compare PBT with 3DCRT and IMRT using validated instruments (Prostate Cancer Symptom Indices scale and the Expanded Prostate Cancer Index Composite) to assess patient-reported bowel and urinary toxicity. ${ }^{29}$ At the first posttreatment follow-up, only patients who had received 3DCRT and IMRT reported a clinically meaningful decrement in bowel QoL. By 12 months and 24 months, all three cohorts reported clinically meaningful decrements in bowel QoL. None of the three cohorts reported a decline in urinary QoL at 24 months. Likewise, in a retrospective analysis by Yu et al, although PBT was associated with a statistically significant reduction in GU toxicity at 6 months compared with IMRT (5.9\% vs 9.5\%, odds ratio [OR] 0.6, 95\% confidence interval $[\mathrm{CI}] 0.38-0.96 ; P=0.03)$, at 12 months posttreatment there was no difference in GU toxicity (18.8\% vs $17.5 \%$, OR $1.08,95 \%$ CI $0.76-1.54 ; P=0.66$ ). There was no statistically significant difference in GI or other toxicity at 6 months or 12 months posttreatment. ${ }^{65}$ Sheets et al, Yu et al, and Kim et al drew from the same combined Surveillance Epidemiology and End Results Medicare database. 2,65,66 Kim et al evaluated GI toxicity outcomes in men with T1-T2 prostate cancer undergoing PBT, IMRT, or 3DCRT. ${ }^{66} \mathrm{As}$ expected, IMRT outperformed 3DCRT in terms of GI toxicity (hazard ratio $0.67,95 \%$ CI $0.55-0.82$ ). What was surprising was that IMRT resulted in a significantly reduced incidence of late bowel toxicity, as compared with PBT (hazard ratio 3.32, 95\% CI, 2.12-5.2). Sheets et al confirmed, although with different risk estimates, Kim et al's results in a propensity score-matched comparison between IMRT and PBT $(n=1,368)$ that IMRT patients had a lower rate of GI toxicities (absolute risk 12.2 vs 17.8 per 100 person-years, relative risk 0.66 ; 95\% CI $0.55-0.79$ ). There were no significant differences in rates of other toxicities. ${ }^{2,66}$

These results of higher rates of rectal toxicity with PBT could be attributed to dosimetric compensations made to ensure adequate coverage at the end of the beam range, or because of the unique biology of the proton beam. Although the RBE of a proton beam is estimated to be 1.1, it might vary depending upon fractionation size, tissue density, and the distribution of proton Bragg peaks within the target. During dose escalation, minute differences in RBE estimates can have a large impact on toxicity.

There are concerns regarding secondary malignancies developing post-RT. This is especially significant, as population based studies have demonstrated an increased risk of several cancers, most notably rectal and bladder cancer, in patients who undergo EBRT for prostate cancer. ${ }^{67}$ Although PBT results in a lower radiation dose to surrounding normal 
tissues, thus reducing the risk of secondary malignancies, the increased scatter from neutrons that arises from the heavier proton particle may increase the risk of secondary malignancies. In spite of the unavailability of populationbased studies, the current literature appears to favor PBT. Fontenot et al used risk modeling to compare IMRT and PBT plans and estimated that PBT reduced the risk of secondary neoplasms by $26 \%-39 \%{ }^{28}$ In a retrospective cohort study matching 558 proton patients with 558 photon patients, after adjusting for sex, age at treatment, primary site, and year of diagnosis, PBT was not associated with an increased risk of secondary malignancy (adjusted hazard ratio 0.52 , $95 \%$ CI $0.32-0.85 ; P=0.009){ }^{68}$

Organs directly irradiated have the greatest risks of secondary malignancies for both modalities. However, this risk can be reduced, as PBT allows significant sparing of portions of the adjacent rectum and bladder. Advanced PBT techniques like PBS have greater dose conformality, and may further decrease the risk of secondary malignancies. However, the relationship between normal tissue dose and secondary malignancy risk is still not fully understood. Therefore, should a decrease in secondary malignancy risk exist with PBT, this benefit is perhaps more clinically significant for younger patients.

Treatment decisions for early stage prostate cancer are fraught with the sense of having to choose between QoL and longevity, although each outcome remains unclear. ${ }^{69}$ This further exacerbates the psychological impact of treatment toxicities and QoL. ${ }^{69,70}$ The risks of poor urinary voiding, nocturia, poor bowel function, fatigue, sexuality, and pain are dependent on factors of technique and total dose delivered. ${ }^{71,72}$ Therefore, with results suggesting that PBT allows better toxicity outcomes, this could potentially alleviate the psychological impact of toxicities and improve QoL for these patients.

\section{Cost concerns}

The significant cost in constructing and maintaining a PBT center has fueled great controversy. Many cost-effectiveness studies have been conducted. The conclusions derived were conflicting. These studies were limited by uncertainties in estimating both cost and benefit. In an estimation by the Institute of Clinical and Economic Review (Boston, MA), the lifetime costs and quality-adjusted life expectancy for IMRT were US\$45,591 and 13.81 years and $\$ 72,789$ and 13.7 years for PBT. ${ }^{8}$ Konski et al published their Markov-model analysis to assess the cost difference per quality-adjusted life-year (QALY) of 91.8 GyE of PBT versus 81 Gy of IMRT. ${ }^{9}$ It was optimistically assumed that a higher dose would confer a $10 \%$ 5 -year freedom from biochemical failure advantage (93\% vs $83 \%$ ) with similar toxicity. ${ }^{55}$ The probable incremental cost-effectiveness ratio for PBT over IMRT was calculated to be $\$ 63,578$ per QALY for a 70 -year-old man and $\$ 55,726$ per QALY for a 60-year-old man. Further adding to this uncertainty is the fact that 91.8 Gy has not been used clinically and the precise incremental increase in cost for PBT varies. Even when using the commonly accepted arbitrary standard of $\$ 50,000$ per QALY, PBT did not appear to be cost-effective. ${ }^{9}$

In contrast, Lundkvist et al reviewed the potential clinical advantages and cost-effectiveness of PBT for prostate cancer using different assumptions (assuming a 20\% reduction in cancer recurrence for PBT, cancer-specific mortality, and a 0.6 relative risk of adverse events compared with conventional radiation) and cost modeling, and estimated a cost of $€ 26,800$ (approximately US\$29,000) per QALY, which would fall well within the standard of US\$50,000 per QALY. ${ }^{10}$

Proponents of PBT argue that two emerging issues may increase the cost-effectiveness of PBT for prostate cancer patients. First, as PBT technology matures and efficiency in delivery increases, costs will become more manageable, as is true with most technologies. Furthermore, PBT may be more cost-effective in the long run, as cyclotrons have a much longer life span than a typical linear accelerator. Second, PBT may become less expensive with a hypofractionated regime (higher daily doses over a shorter time period). To date, published early results of several recent trials have suggested safety and equivalence between conventionally fractionated regimens, typically involving 8-9 weeks of treatment, and hypofractionated courses, which are delivered using higher daily doses over a shorter time period. ${ }^{73-76}$ A Phase III prospective randomized trial of standard fractionation (44 PBT fractions [1.8 Gy RBE $\times 44]$ ) vs hypofractionation (five PBT fractions [7.6 Gy RBE $\times 5$ ]) with proton RT for low-risk adenocarcinoma of the prostate (GU002-10/NCT01230866) is currently open. ${ }^{77}$ This has the potential of significantly reducing PBT cost if the five-fraction treatment arm achieves similar or better treatment outcome than the 44-fraction treatment arm.

To summarize, PBT presents a significant theoretical dosimetric advantage over photon EBRT. Improvements in targeting and dose delivery resulted in improved diseasecontrol rates for patients receiving photon- or proton-based treatment. However, it remains challenging to compare toxicities and efficacy-outcome end points directly across the 
literature, due to differences among patient characteristics, treatment techniques, and outcome measures. To date, current photon- and proton-based treatments have been proven to be effective within a well-defined toxicity profile. However, it remains unclear if one modality is superior to another. The current literature, as discussed earlier, suggests comparable tumor control with no clear advantage in terms of late effects and a controversial suggestion that protons may be associated with a higher incidence of late bowel toxicity. It is with hope that ongoing trials ${ }^{77-79}$ can better elucidate which technique confers a superior advantage for patients.

\section{Future directions}

The results reported in the literature are those of the old technology of passive scattering. PBS, as mentioned earlier, is now available as an advanced technology, and will likely replace the scattered beam. Utilizing PBS-PBT allows the delivery of a sequence of fluences of differing proton energies that yields a more conformal dose distribution to the target with sharp dose gradients that avoids adjacent OARs. This is akin to photon IMRT and is known as IMPT. Studies suggest further dosimetric and clinical advantage with IMPT for prostate treatment and in unique clinical situations, such as treating recurrent prostate cancers. ${ }^{80-84}$ Many investigational advances, such as utilizing novel angles, ${ }^{85}$ partial prostate therapy, and focal dose boosting in regions of heavy tumor burden, ${ }^{4}$ are promising approaches that have yet to be utilized in the clinical setting.

\section{Conclusion}

The unique physical properties of protons allow dose escalation to the prostate while sparing adjacent proximal OARs. Theoretically, this should confer superior disease control while reducing toxicity and second malignancies. This confers a dosimetric advantage over photon IMRT, and makes using PBT as a source of therapeutic radiation attractive. The current literature, consisting of prospective Phase II trials and retrospective studies, has demonstrated favorable outcomes for early stage prostate cancer utilizing PBT. PBT is significantly expensive, and patients would have to absorb much of this cost. This remains a barrier to its widespread use. To date, the costeffectiveness of PBT remains conflicting. Its cost-effectiveness requires more study. More studies are required for a better estimation. Ongoing trials directly comparing PBT with photon EBRT will hopefully elucidate the value of PBT for early stage prostate cancer. Unless there is overwhelming evidence for the clinical superiority of PBT over present advanced techniques, the case for PBT remains questionable.

\section{Disclosure}

The authors report no conflicts of interest in this work.

\section{References}

1. Aral IA. External beam radiotherapy in prostate cancer. 2015. Available from: http://emedicine.medscape.com/article/454283-overview. Accessed March 9, 2016.

2. Sheets NC, Goldin GH, Meyer AM, et al. Intensity-modulated radiation therapy, proton therapy, or conformal radiation therapy and morbidity and disease control in localized prostate cancer. JAMA. 2012;307(15): 1611-1620.

3. Novaes P, Mottas R, Lundgren M. Treatment of prostate cancer with intensity modulated radiation therapy (IMRT). Rev Assoc Med Bras. 2015;61(1):8-16.

4. Efstathiou J, Trofimov A, Zietman A. Life, liberty, and the pursuit of protons: an evidence-based review of the role of particle therapy in the treatment of prostate cancer. Cancer J. 2009;15(4):312-318.

5. Trofimov A, Nguyen P, Coen J, et al. Radiotherapy treatment of earlystage prostate cancer with IMRT and protons: a treatment planning comparison. Int J Radiat Oncol Biol Phys. 2007;69(2):444-453.

6. Vargas C, Fryer A, Mahajan C, et al. Dose-volume comparison of proton therapy and intensity-modulated radiotherapy for prostate cancer. Int $J$ Radiat Oncol Biol Phys. 2008;70(3):744-751.

7. Zhang X, Dong L, Lee A, et al. Effect of anatomic motion on proton therapy dose distributions in prostate cancer treatment. Int $J$ Radiat Oncol Biol Phys. 2007;67(2):620-629.

8. Kagan A, Schulz R. Proton-beam therapy for prostate cancer. Cancer J. 2010;16(5):405-409.

9. Konski A, Speier W, Hanlon A, Beck J, Pollack A. Is proton beam therapy cost effective in the treatment of adenocarcinoma of the prostate? J Clin Oncol. 2007;25(24):3603-3608.

10. Lundkvist J, Ekman M, Ericsson S, Jönsson B, Glimelius B. Proton therapy of cancer: potential clinical advantages and cost-effectiveness. Acta Oncol. 2005;44(8):850-861.

11. American Cancer Society. External beam radiation. 2014. Available from: http://www.cancer.org/treatment/treatmentsandsideeffects/treatmenttypes/radiation/radiationtherapyprinciples/radiation-therapy-principleshow-is-radiation-given-external-beam-rad. Accessed March 9, 2016.

12. Campeau F, Phelps J. Limited Radiography. Albany (NY): Delmar; 1992.

13. Proton Therapy Center. Principles of proton therapy. Available from: http://www.proton-cancer-treatment.com/proton-therapy/principles-ofproton-therapy. Accessed March 9, 2016.

14. Wedenberg M, Lind B, Hårdemark B. A model for the relative biological effectiveness of protons: the tissue specific parameter $\alpha / \beta$ of photons is a predictor for the sensitivity to LET changes. Acta Oncol. 2012;52(3):580-588.

15. Raaymakers BW, Lagendijk JJ, van Vulpen M, Hartman J. MRI guided proton therapy: integrating MRI functionality with a proton therapy system. Available from: http://www.radiotherapie.nl/research/researchprojects/mri-guided-radiotherapy/mri-guided-proton-therapy. Accessed March 9, 2016.

16. Halperin E, Perez C, Brady L. Perez and Brady's Principles and Practice of Radiation Oncology. 5th ed. Philadelphia: Lippincott Williams \& Wilkins; 2007.

17. MD Anderson Cancer Center. Proton therapy for prostate cancer. Available from: https://www.mdanderson.org/patients-family/diagnosis-treatment/care-centers-clinics/proton-therapy-center/conditions-wetreat/prostate-cancer.html. Accessed March 9, 2016.

18. Bortfeld T, Paganetti H, Kooy H. MO-A-T-6B-01: proton beam radiotherapy - the state of the art. Med Phys. 2005;32(6):2048.

19. Bekelman J, Mitra N, Efstathiou J, et al. Outcomes after intensitymodulated versus conformal radiotherapy in older men with nonmetastatic prostate cancer. Int J Radiat Oncol Biol Phys. 2011;81(4): e325-e334. 
20. Dinan M, Robinson T, Zagar T, et al. Changes in initial treatment for prostate cancer among Medicare beneficiaries, 1999-2007. Int J Radiat Oncol Biol Phys. 2012;82(5):e781-e786.

21. Segal R, Reid R, Courneya K, et al. Randomized controlled trial of resistance or aerobic exercise in men receiving radiation therapy for prostate cancer. J Clin Oncol. 2008;27(3):344-351.

22. Pickles T, Graham P. What happens to testosterone after prostate radiation monotherapy and does it matter? J Urol. 2002;167(6):2448-2452.

23. Zagars G, Pollack A. Serum testosterone levels after external beam radiation for clinically localized prostate cancer. Int J Radiat Oncol Biol Phys. 1997;39(1):85-89.

24. Nichols R, Morris C, Hoppe B, et al. Proton radiotherapy for prostate cancer is not associated with post-treatment testosterone suppression. Int J Radiat Oncol Biol Phys. 2012;82(3):1222-1226.

25. Kil W, Nichols R, Hoppe B, et al. Hypofractionated passively scattered proton radiotherapy for low- and intermediate-risk prostate cancer is not associated with post-treatment testosterone suppression. Acta Oncolog. 2013;52(3):492-497.

26. Rechner L, Howell R, Zhang R, Etzel C, Lee A, Newhauser W. Risk of radiogenic second cancers following volumetric modulated arc therapy and proton arc therapy for prostate cancer. Phys Med Biol. 2012;57(21): 7117-7132.

27. Yoon M, Ahn S, Kim J, et al. Radiation-induced cancers from modern radiotherapy techniques: intensity-modulated radiotherapy versus proton therapy. Int J Radiat Oncol Biol Phys. 2010;77(5): 1477-1485.

28. Fontenot J, Lee A, Newhauser W. Risk of secondary malignant neoplasms from proton therapy and intensity-modulated X-ray therapy for early-stage prostate cancer. Int J Radiat Oncol Biol Phys. 2009;74(2): 616-622.

29. Gray P, Paly J, Yeap B, et al. Patient-reported outcomes after 3dimensional conformal, intensity-modulated, or proton beam radiotherapy for localized prostate cancer. Cancer. 2013;119(9):1729-1735.

30. Fiorino C, Fellin G, Rancati T, et al. Clinical and dosimetric predictors of late rectal syndrome after 3D-CRT for localized prostate cancer: preliminary results of a multicenter prospective study. Int J Radiat Oncol Biol Phys. 2008;70(4):1130-1137.

31. Jackson A, Skwarchuk M, Zelefsky M, et al. Late rectal bleeding after conformal radiotherapy of prostate cancer (II): volume effects and dose-volume histograms. Int J Radiat Oncol Biol Phys. 2001;49(3):685-698.

32. Karlsdóttir A, Muren L, Wentzel-Larsen T, Dahl O. Late gastrointestinal morbidity after three-dimensional conformal radiation therapy for prostate cancer fades with time in contrast to genitourinary morbidity. Int J Radiat Oncol Biol Phys. 2008;70(5):1478-1486.

33. Nguyen P, Chen R, Hoffman K, et al. Rectal dose-volume histogram parameters are associated with long-term patient-reported gastrointestinal quality of life after conventional and high-dose radiation for prostate cancer: a subgroup analysis of a randomized trial. Int J Radiat Oncol Biol Phys. 2010;78(4):1081-1085.

34. Skwarchuk M, Jackson A, Zelefsky M, et al. Late rectal toxicity after conformal radiotherapy of prostate cancer (I): multivariate analysis and dose-response. Int J Radiat Oncol Biol Phys. 2000;47(1): 103-113.

35. Tucker S, Dong L, Michalski J, et al. Do intermediate radiation doses contribute to late rectal toxicity? An analysis of data from Radiation Therapy Oncology Group protocol 94-06. Int J Radiat Oncol Biol Phys. 2012;84(2):390-395.

36. Goitein M. Magical protons? Int J Radiat Oncol Biol Phys. 2008; 70(3):654-656.

37. Clasie B, Wroe A, Kooy H, et al. Assessment of out-of-field absorbed dose and equivalent dose in proton fields. Med Phys. 2010;37(1): 311-321.

38. $\mathrm{Lu} \mathrm{H}$. A potential method for in vivo range verification in proton therapy treatment. Phys Med Biol. 2008;53(5):1413-1424.

39. Yoon M, Kim D, Shin D, et al. Inter- and intrafractional movementinduced dose reduction of prostate target volume in proton beam treatment. Int J Radiat Oncol Biol Phys. 2008;71(4):1091-1102.
40. Yoon M, Shin D, Kwak J, et al. Characteristics of movement-induced dose reduction in target volume: a comparison between photon and proton beam treatment. Med Dosim. 2009;34(3):191-201.

41. Trofimov A, Nguyen P, Efstathiou J, et al. Interfractional variations in the setup of pelvic bony anatomy and soft tissue, and their implications on the delivery of proton therapy for localized prostate cancer. Int $J$ Radiat Oncol Biol Phys. 2011;80(3):928-937.

42. Wang Y, Efstathiou J, Sharp G, Lu H, Ciernik IF, Trofimov A. Evaluation of the dosimetric impact of interfractional anatomical variations on prostate proton therapy using daily in-room CT images. Med Phys. 2011;38(8):4623-4633.

43. Tewari A. Prostate Cancer. London: Springer; 2013.

44. Athar B, Bednarz B, Seco J, Hancox C, Paganetti H. Comparison of out-of-field photon doses in 6 MV IMRT and neutron doses in proton therapy for adult and pediatric patients. Phys Med Biol. 2010;55(10): 2879-2891.

45. Robertson J, Williams J, Schmidt R, Little J, Flynn D, Suit H. Radiobiological studies of a high-energy modulated proton beam utilizing cultured mammalian cells. Cancer. 1975;35(6):1664-1677.

46. Budäus L, Bolla M, Bossi A, et al. Functional outcomes and complications following radiation therapy for prostate cancer: a critical analysis of the literature. Eur Urol. 2012;61(1):112-127.

47. Slater J, Rossi C, Yonemoto L, et al. Proton therapy for prostate cancer: the initial Loma Linda University experience. Int J Radiat Oncol Biol Phys. 2004;59(2):348-352.

48. Mendenhall N, Li Z, Hoppe B, et al. Early outcomes from three prospective trials of image-guided proton therapy for prostate cancer. Int J Radiat Oncol Biol Phys. 2012;82(1):213-221.

49. Vora S, Wong W, Schild S, Ezzell G, Halyard M. Analysis of biochemical control and prognostic factors in patients treated with either low-dose three-dimensional conformal radiation therapy or high-dose intensity-modulated radiotherapy for localized prostate cancer. Int $J$ Radiat Oncol Biol Phys. 2007;68(4):1053-1058.

50. Zietman A, Bae K, Slater J, et al. Randomized trial comparing conventional-dose with high-dose conformal radiation therapy in earlystage adenocarcinoma of the prostate: long-term results from Proton Radiation Oncology Group/American College of Radiology 95-09. $J$ Clin Oncol. 2010;28(7):1106-1111.

51. Johansson S, Aström L, Sandin F, Isacsson U, Montelius A, Turesson I. Hypofractionated proton boost combined with external beam radiotherapy for treatment of localized prostate cancer. Prostate Cancer. 2012;2012:654861.

52. Nihei K. Phase II feasibility study of high-dose radiotherapy for prostate cancer using proton boost therapy: first clinical trial of proton beam therapy for prostate cancer in Japan. Jpn J Clin Oncol. 2005;35(12):745-752.

53. Talcott J. Patient-reported long-term outcomes after conventional and high-dose combined proton and photon radiation for early prostate cancer. JAMA. 2010;303(11):1046-1053.

54. Zietman A, DeSilvio M, Slater J, et al. Comparison of conventionaldose vs high-dose conformal radiation therapy in clinically localized adenocarcinoma of the prostate. JAMA. 2005;294(10):1233-1239.

55. Coen J, Bae K, Zietman A, et al. Acute and late toxicity after dose escalation to $82 \mathrm{GyE}$ using conformal proton radiation for localized prostate cancer: initial report of American College of Radiology phase II study 03-12. Int J Radiat Oncol Biol Phys. 2011;81(4):1005-1009.

56. Peeters S. Dose-Response in radiotherapy for localized prostate cancer: results of the Dutch multicenter randomized phase III trial comparing 68 Gy of radiotherapy with 78 Gy. J Clin Oncol. 2006;24(13): 1990-1996.

57. Dearnaley D, Sydes M, Graham J, et al. Escalated-dose versus standarddose conformal radiotherapy in prostate cancer: first results from the MRC RT01 randomised controlled trial. Lancet Oncol. 2007;8(6): 475-487.

58. Al-Mamgani A, van Putten W, Heemsbergen W, et al. Update of Dutch multicenter dose-escalation trial of radiotherapy for localized prostate cancer. Int J Radiat Oncol Biol Phys. 2008;72(4):980-988. 
59. Kuban D, Tucker S, Dong L, et al. Long-term results of the M. D. Anderson Randomized dose-escalation trial for prostate cancer. Int $J$ Radiat Oncol Biol Phys. 2008;70(1):67-74.

60. Syndikus I, Morgan R, Sydes M, Graham J, Dearnaley D. Late gastrointestinal toxicity after dose-escalated conformal radiotherapy for early prostate cancer: results from the UK Medical Research Council RT01 trial (ISRCTN47772397). Int J Radiat Oncol Biol Phys. 2010;77(3):773-783.

61. Beckendorf V, Guerif S, Le Prisé E, et al. 70 Gy versus 80 Gy in localized prostate cancer: 5 -year results of GETUG 06 randomized trial. Int J Radiat Oncol Biol Phys. 2011;80(4):1056-1063.

62. Nihei K, Ogino T, Onozawa M, et al. Multi-institutional phase II study of proton beam therapy for organ-confined prostate cancer focusing on the incidence of late rectal toxicities. Int J Radiat Oncol Biol Phys. 2011;81(2):390-396.

63. Mendenhall N, Li Z, Hoppe B, et al. Early outcomes from three prospective trials of image-guided proton therapy for prostate cancer. Int J Radiat Oncol Biol Phys. 2012;82(1):213-221.

64. Coen J, Paly J, Niemierko A, et al. Long-term quality of life outcome after proton beam monotherapy for localized prostate cancer. Int $J$ Radiat Oncol Biol Phys. 2012;82(2):e201-e209.

65. Yu J, Soulos P, Herrin J, et al. Proton radiation therapy for prostate cancer in the Medicare population: patterns of care and comparison of early toxicity with intensity modulated radiation therapy. Int J Radiat Oncol Biol Phys. 2012;84(3):S13.

66. Kim S, Shen S, Moore D, et al. Late gastrointestinal toxicities following radiation therapy for prostate cancer. Eur Urol. 2011;60(5):908-916.

67. Moon K, Stukenborg G, Keim J, Theodorescu D. Cancer incidence after localized therapy for prostate cancer. Cancer. 2006;107(5):991-998.

68. Chung C, Yock T, Nelson K, Xu Y, Keating N, Tarbell N. Incidence of second malignancies among patients treated with proton versus photon radiation. Int J Radiat Oncol Biol Phys. 2013;87(1):46-52.

69. Chen R, Clark J, Manola J, Talcott J. Treatment 'mismatch' in early prostate cancer. Cancer. 2007;112(1):61-68.

70. Namiki S, Arai Y. Health-related quality of life in men with localized prostate cancer. Int J Urol. 2010;17(2):125-138.

71. Namiki S, Takegami M, Ishidoya S, Numata I, Arai Y. Impact of nocturia on disease-specific quality of life for men with localized prostate cancer. Qual Life Res. 2011;20(10):1609-1615.

72. Eton D, Lepore S. Prostate cancer and health-related quality of life: a review of the literature. Psychooncology. 2002;11(4):307-326.

73. Arcangeli S, Strigari L, Gomellini S, et al. Updated results and patterns of failure in a randomized hypofractionation trial for high-risk prostate cancer. Int J Radiat Oncol Biol Phys. 2012;84(5):1172-1178.
74. Boike T, Lotan Y, Cho L, et al. Phase I dose-escalation study of stereotactic body radiation therapy for low- and intermediate-risk prostate cancer. J Clin Oncol. 2011;29(15):2020-2026.

75. Dearnaley D, Syndikus I, Sumo G, et al. Conventional versus hypofractionated high-dose intensity-modulated radiotherapy for prostate cancer: preliminary safety results from the CHHiP randomised controlled trial. Lancet Oncol. 2012;13(1):43-54.

76. Kupelian P, Willoughby T, Reddy C, Klein E, Mahadevan A. Hypofractionated intensity-modulated radiotherapy (70 Gy at 2.5 Gy per fraction) for localized prostate cancer: Cleveland Clinic experience. Int J Radiat Oncol Biol Phys. 2007;68(5):1424-1430.

77. Proton Collaborative Group. Study of hypo-fractionated proton radiation for low risk prostate cancer. Available from: https://clinicaltrials.gov/ ct2/show/NCT01230866. NLM identifier: NCT01230866. Accessed March 12, 2016.

78. MD Anderson Cancer Center. Hypofractionated proton beam therapy for localized prostate cancer. Available from: https://clinicaltrials.gov/ ct2/show/NCT01950351. NLM identifier: NCT01950351. Accessed March 12, 2016.

79. Massachusetts General Hospital. Proton therapy vs. IMRT for low or intermediate risk prostate cancer. Available from: https://clinicaltrials.gov/ct2/show/NCT01617161. NLM identifier: NCT01617161. Accessed March 12, 2016.

80. Kase Y, Yamashita H, Fuji H, et al. A treatment planning comparison of passive-scattering and intensity-modulated proton therapy for typical tumor sites. J Radiat Res. 2012;53(2):272-280.

81. Widesott L, Pierelli A, Fiorino C, et al. Helical tomotherapy vs. intensity-modulated proton therapy for whole pelvis irradiation in highrisk prostate cancer patients: dosimetric, normal tissue complication probability, and generalized equivalent uniform dose analysis. Int $J$ Radiat Oncol Biol Phys. 2011;80(5):1589-1600.

82. Weber D, Wang H, Cozzi L, et al. RapidArc, intensity modulated photon and proton techniques for recurrent prostate cancer in previously irradiated patients: a treatment planning comparison study. Radiat Oncol. 2009;4:34.

83. Lomax A, Pedroni E, Rutz H, Goitein G. The clinical potential of intensity modulated proton therapy. Z Med Phys. 2004;14(3):147-152.

84. Kagan A, Yeh J, Schulz R. Is proton-beam therapy better than intensitymodulated radiation therapy for prostate cancer? Am J Clin Oncol. 2014;37(6):525-527.

85. Tang $\mathrm{S}$, Both $\mathrm{S}$, Bentefour $\mathrm{H}$, et al. Improvement of prostate treatment by anterior proton fields. Int J Radiat Oncol Biol Phys. 2012;83(1): 408-418.
OncoTargets and Therapy

\section{Publish your work in this journal}

OncoTargets and Therapy is an international, peer-reviewed, open access journal focusing on the pathological basis of all cancers, potential targets for therapy and treatment protocols employed to improve the management of cancer patients. The journal also focuses on the impact of management programs and new therapeutic agents and protocols on

\section{Dovepress}

patient perspectives such as quality of life, adherence and satisfaction The manuscript management system is completely online and includes a very quick and fair peer-review system, which is all easy to use. Visit http://www.dovepress.com/testimonials.php to read real quotes from published authors. 